\title{
Unveiling the massive stars in the Galactic center
}

\author{
H. Dong ${ }^{1}$, J. Mauerhan ${ }^{2,3}$, M. R. Morris ${ }^{4}$, Q. D. Wang ${ }^{5}$ and \\ A. Cotera $^{6}$ \\ ${ }^{1}$ National Optical Astronomy Observatory, Tucson, AZ 85719 \\ ${ }^{2}$ Department of Physics, University of California, Berkeley, CA 94720 \\ ${ }^{3}$ Steward Observatory, University of Arizona, Tucson, AZ 85719 \\ ${ }^{4}$ Department of Physics and Astronomy, University of California, Los Angeles, CA 90095 \\ ${ }^{5}$ Department of Astronomy, University of Massachusetts, Amherst, MA 01003 \\ ${ }^{6}$ SETI Institute, Mountain View, CA 94043 \\ email: hdong@noao.edu
}

\begin{abstract}
We present our recent efforts to unveil and understand the origin of massive stars outside the three massive star clusters in the Galactic center. From our Hubble/NICMOS survey of the Galactic center, we have identified 180 Paschen- $\alpha$ emitting sources, most of which should be evolved massive stars with strong optically thin stellar winds. Recently, we obtained Gemini GNIRS/NIFS $H$ - and $K$-band spectra of eight massive stars near the Arches cluster. From their radial velocities, ages and masses, we suggest that in our sample, two stars are previous members of the Arches cluster, while other two stars embedded in the H1/H2 HiI regions formed in-situ.
\end{abstract}

Keywords. Massive Stars, HiI regions, star formation

\section{Motivation}

The Galactic center (GC) is the only galactic nucleus in which we can study the coevolution of nuclear star formation activity, a nuclear star cluster and a supermassive black hole $(\mathrm{SMBH})$ in great detail. During the last two decades, a SMBH, Sgr A* (Ghez et al. 2008, Gillessen et al. 2009), and a nuclear cluster, the Central cluster (Genzel et al. 2003), are indeed found in the GC. Meanwhile, the central $300 \mathrm{pc}$ of the GC, known as, the central molecular zone $(\mathrm{CMZ})$, includes $\sim 4 \times 10^{7} \mathrm{M}_{\odot}$ molecular clouds (Morris \& Serabyn 1996, Molinari et al. 2011), which are forming stars with a rate of about $0.03 \mathrm{M}_{\odot} \mathrm{yr}^{-1}$ (Longmore et al. 2013). Because of the proximity, we can detect individual massive stars and study their interaction with the interstellar medium (ISM).

One important question that we want to address is how many stars formed during the last $10 \mathrm{Myr}$ in the CMZ and whether the energy released by these stars could explain the Fermi bubbles ( $\mathrm{Su}$ et al. 2010, Crocker \& Aharonian 2011). Only three young, massive and compact star clusters, the Arches, Quintuplet and Central clusters (2-7 Myr old, Figer et al. 1999, Figer et al. 2002, Genzel et al. 2003), have been identified in groundbased near-infrared (NIR) surveys of the Galactic center. Young massive stars outside of these three clusters have largely been unknown. The slow progress in detecting these 'field' stars is largely owed to the lack of an efficient way to distinguish young massive stars and evolved low-mass stars through their NIR colors.

Our Hubble/NICMOS Paschen- $\alpha$ GC survey (Wang et al. 2010) provides a systematic way to identify evolved massive stars (EMS) having strong stellar winds. We mapped out the central $39^{\prime \prime} \times 15^{\prime \prime}$ of the GC with NIC3 in two narrow-band filters: F187N (on-line, 1.87 $\mu \mathrm{m}$ ) and F190N (adjacent continuum, $1.90 \mu \mathrm{m}$ ) (Dong et al. 2011). Combined with archived Hubble/NICMOS NIC2 observations of the three star clusters 

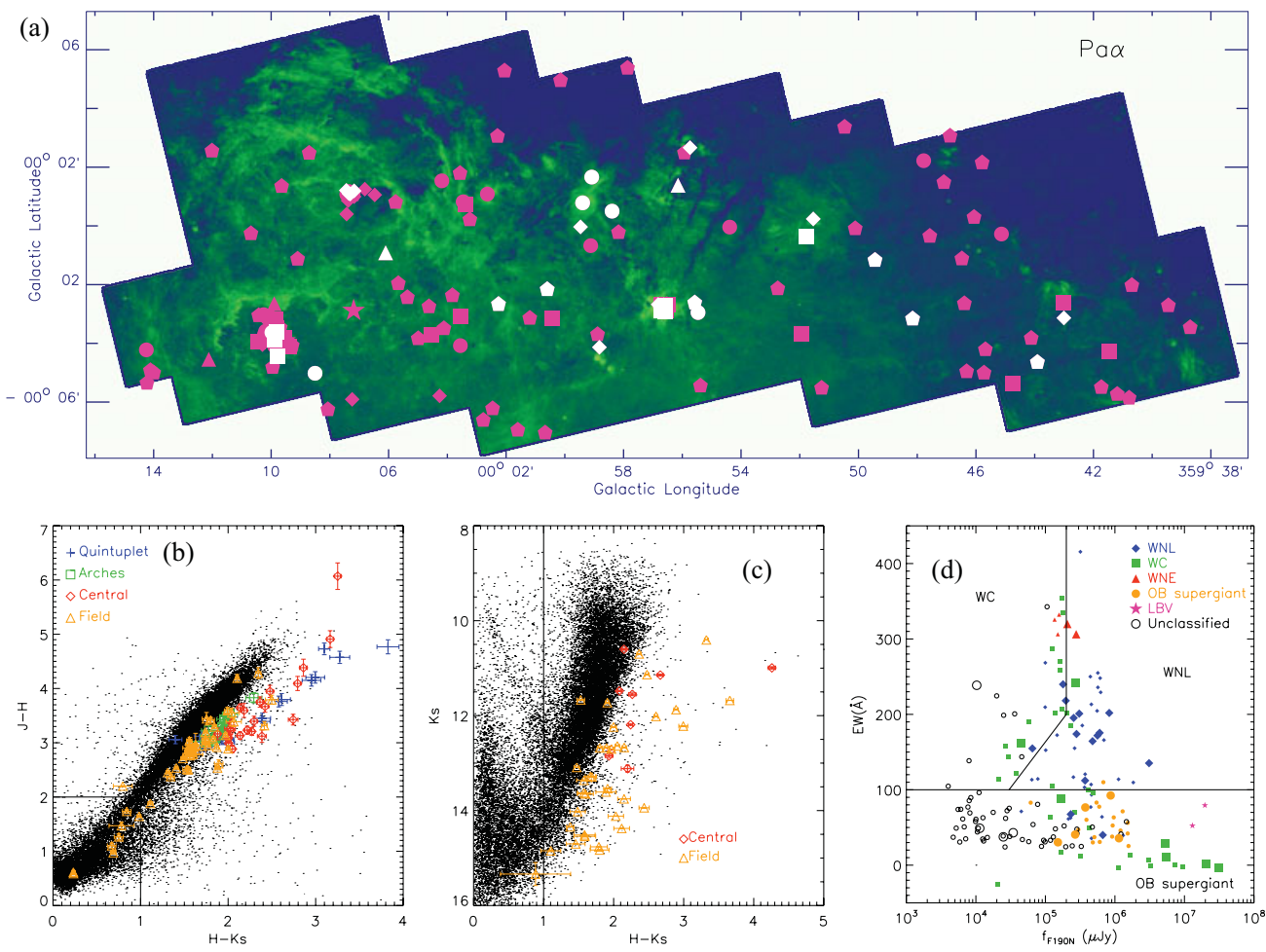

Figure 1. (a) 180 PESs overlaid on the mosaic image of Paschen- $\alpha$ intensity ('diamond': WNL stars, 'square': WC stars, 'triangle': WNE stars, 'circle': OB supergiants, 'star symbols': LBV stars). The 'pentagon' symbols represent those Paschen-alpha without available spectroscopic identifications. The filled symbols indicate ones with X-ray counterparts. (b) and (c) are infrared color-color and magnitude-color diagram of 119 and 45 PESs, compared to the field stars from the SIRIUS survey (black small dots). Thick black lines delineate the region occupied by foreground stars $(H-K<1$ and $J-H<2)$. (d) The combined Paschen- $\alpha$ EW vs. $f_{F 190 N}^{o}$ plot of stars of various types. Lines are drawn to roughly show the regions dominated by WNL, $\mathrm{WC}$ and $\mathrm{OB}$ supergiants. The stars with larger symbols are the ones with X-ray counterparts. [A COLOR VERSION IS AVAILABLE ONLINE.]

at the same bands, we identify 180 Paschen- $\alpha$ emitting sources (PESs) in our survey area (see Figure 1a), which have strong line emission in the F187N band and could be EMSs (Dong et al. 2012). Half of these stars lie outside the three clusters and are scattered throughout the GC. Unlike those in the clusters, most of these 'field' stars still don't have available spectroscopic identifications.

\section{Near-infrared photometry of Paschen- $\alpha$ emitting sources}

Before obtaining spectroscopic observations of 'field' PESs, we use the existing NIR photometry to study their properties. We employ the SIRIUS (Nagayama et al. 2003) and 2MASS (Skrutskie et al. 2006) source catalogs. Figure 1b and 1c show the color-color and magnitude-color diagrams for our 164 Paschen- $\alpha$ emitting sources with available NIR photometry. Most of our PESs are very red and indeed within the GC. We also estimate their extinction using the NIR colors (Dong et al. 2012).

Figure 1d shows the equivalent width (EW) in the $\mathrm{F} 187 \mathrm{~N}$ band versus the intrinsic F190N intensity of our PESs. We empirically divide this figure into three parts according 
to their spectral types. The EWs of the WN stars are large and seem to be correlated with the intrinsic F190N intensity. On the other hand, the EWs of the WC stars span a large range, which could be due to surrounding hot dust shells enhancing the F187N continuum and thus reducing the EWs. Compared to the WN stars, the EWs of OB supergiants are smaller, indicating weaker stellar winds. Therefore, those PESs without available spectroscopic identification most likely represent a combination of WC stars and OB supergiants.

\section{The origin of Paschen- $\alpha$ emitting sources}

These 'field' PESs could: 1) be the leftover of dissolved star clusters; 2) be ejected out of the three existing massive star clusters through three-body interactions; and/or 3) form in-situ.

In order to understand their origin, we measured the radial velocities of eight $\mathrm{OB}$ supergiants and $\mathrm{O} \mathrm{If}^{+}$stars within $10^{\prime}$ of the Arches cluster with Gemini GNIRS/NIFS NIR spectra. With the Gemini/GNIRS long-slit spectra, we also detects the $\operatorname{Br} \gamma$ line emission from nearby HII regions. We further complement our data with previous radio H92 $\alpha$ (Lang et al. 2002) and CS observations (Tsuboi et al. 1999) for the radial velocity of nearby ionized gas and molecular clouds. By comparing their unreddened NIR colors with Geneva stellar evolutionary tracks, we conclude that these eight stars are younger than $5 \mathrm{Myr}$ and have masses $>25 \mathrm{M}_{\odot}$. Therefore, they could not be from dissovled clusters, which should have lifetimes greater than 7 Myr (Portegies Zwart et al. 2002).

We mark the locations of the eight stars in Figure 2a. Combined with the proper motion derived from their projected distances, the $3-\mathrm{D}$ velocities of the stars relative to the Arches cluster (assuming that it is their origin) are $60-140 \mathrm{~km} \mathrm{~s}^{-1}$, still within the range predicted by three-body interactions of massive stars (Gvaramadze \& Gualandris 2011). On the other hand, most of these stars are associated with in local extended Paschen- $\alpha$ emission and could form in-situ.

We compare the radial velocities of these stars with those of nearby ionized gas, molecular clouds and the Arches cluster in Figure 2b. All the stars are blueshifted, relative to the Arches cluster, by $>50 \mathrm{~km} \mathrm{~s}^{-1}$. The different radial velocities of P97/P107 and nearby molecular clouds indicate that they are just runaway stars, while near P98/P100, the morphologies of ionized gas don't support that they are young star formation regions. Because P97 and P98 have spectra and ages similar to those of $\mathrm{O} \mathrm{If}^{+}$stars in the Arches cluster, they could be previous members of the Arches cluster. Because P100 and P107 are relatively older than the Arches cluster (Dong et al. in preparation), future proper motion are needed to confirm their relationship. Combined with three WNh stars within 1-2 pc of the Arches cluster suggested by Mauerhan et al. (2010), there are five 'field' EMSs which could have been ejected from the Arches cluster.

A complete census of these deserters is essential to understanding the properties of the Arches cluster. In addition to producing these runaway stars, three-body interactions may also produce tightly bound massive binaries. Several of these binaries probably don't have enough velocities to have escaped from the Arches cluster. Indeed, three massive binaries have been found in the Arches cluster (Wang et al. 2006), through their $\mathrm{X}$-ray emission from the wind collision zones. Of course, there may be more massive binaries in the Arches cluster, which produce too weak X-ray emission to have been detected. If such binaries are considered as single stars, their ages/masses could be underestimated/overestimated. Therefore, detecting the ejected massive stars can provide useful constraints on the dynamic processes of the cluster. 

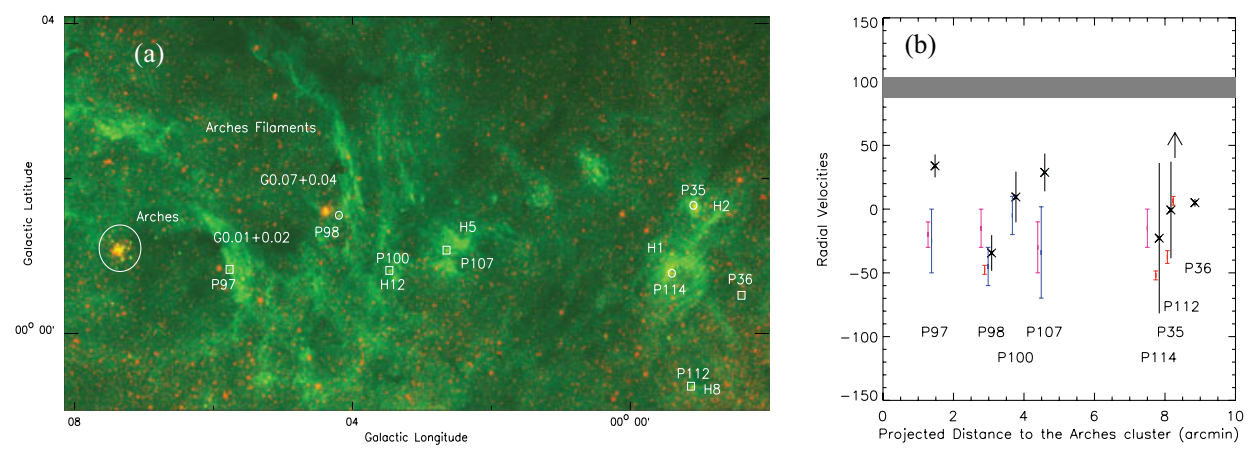

Figure 2. Left: eight evolved massive stars overlaid on the color image; red: Hubble F190N image and green: Paschen- $\alpha$ emission. We mark the location of the Arches cluster and different HII regions. Right: the radial velocities of our massive stars ('crosses'), nearby ionized gas (the blue and red lines on the left side of individual 'crosses') and molecular clouds (the cyan lines). The $\mathrm{x}$-axis is the projected distance from the massive stars to the Arches cluster. The grey region is the radial velocity of the Arches cluster. [A COLOR Version Is AVAILABle OnLINE.]

The remaining four stars could be unrelated to the Arches cluster. No stars in the Arches cluster have spectral types similar to P112, and the $\mathrm{O} \mathrm{If}^{+}$stars in the Arches cluster are more massive than P36. P114 and P36 are embedded in the H1 and H2 HII regions, which have shell-like structures. P36/P114 and the nearby $-30-0 \mathrm{~km} \mathrm{~s}^{-1}$ molecular cloud have similar radial velocities, redshifted in respect to surrounding ionized gas. The lack of no $\mathrm{H}_{2} 2.121 \mu \mathrm{m}$ line indicates that the shell-like structures can't be explained by a bow shock model, but perhaps by a pressure-driven model. Therefore, P36/P112 are not runaway stars and could have formed in-situ. According to the toy model of the molecular clouds in the CMZ proposed by Molinari et al. (2011), about 2 Myr ago, the nearby $-30-0 \mathrm{~km} \mathrm{~s}^{-1}$ molecular cloud could have passed its closest approach to Sgr A* and the star formation was triggered by the strong stellar winds from the Central cluster, and also the possible feedback from Sgr A*.

\section{References}

Ghez, A. M., Salim, S., Weinberg, N. N., et al. 2008, ApJ 689, 1044

Gillessen, S., Eisenhauer, F., Trippe, S., et al. 2009, ApJ 692, 1075

Genzel, R., Schödel, R., Ott, T., et al. 2003, ApJ 594, 812

Morris, M. \& Serabyn, E. 1996, ARAA 34, 645

Molinari, S., Bally, J., Noriega-Crespo, A., et al. 2011, ApJ Lett. 735, L33

Longmore, S. N., Bally, J., Testi, L., et al. 2013, MNRAS 429, 987

Su, M., Slatyer, T. R., \& Finkbeiner, D. P. 2010, ApJ 724, 1044

Crocker, R. M. \& Aharonian, F. 2011, Phys. Rev. Lett. 106, 101102

Figer, D. F., McLean, I. S., \& Morris, M. 1999, ApJ 514, 202

Figer, D. F., Najarro, F., Gilmore, D., et al. 2002, ApJ 581, 258

Wang, Q. D., Dong, H., Cotera, A., et al. 2010, MNRAS 402, 895

Dong, H., Wang, Q. D., Cotera, A., et al. 2011, MNRAS 417, 114

Dong, H., Wang, Q. D., \& Morris, M. R. 2012, MNRAS 425, 884

Nagayama, T., Nagashima, C., Nakajima, Y., Nagata, T., et al., SPIE 4841, 459N

Skrutskie, M. F., Cutri, R. M., Stiening, R., Weinberg, M. D., \& et al., 2006, AJ 131, $1163 \mathrm{~S}$

Lang, C. C., Goss, W. M., \& Morris, M. 2002, AJ 124, 2677 
Tsuboi, M., Handa, T., \& Ukita, N. 1999, ApJS 120, 1

Gvaramadze, V. V. \& Gualandris, A. 2011, MNRAS 410, 304

Portegies Zwart, S. F., Makino, J., McMillan, S. L. W., \& Hut, P. 2002, ApJ 565, 265

Mauerhan, J. C., Cotera, A., Dong, H., \& et al., 2010, ApJ 725, 188

Wang, Q. D., Dong, H., \& Lang, C. 2006, MNRAS 371, 38 